\title{
Electrospray Tandem Mass Spectrometry of Mixed-Sequence RNA/DNA Oligonucleotides
}

\author{
Stefan Schürch, Eloy Bernal-Méndez, and Christian J. Leumann \\ Department of Chemistry and Biochemistry, University of Bern, Bern, Switzerland
}

\begin{abstract}
The fragmentation of electrospray-generated multiply deprotonated RNA and mixed-sequence RNA/DNA pentanucleotides upon low-energy collision-induced dissociation (CID) in a hybrid quadrupole time-of-flight mass spectrometer was investigated. The goal of unambiguous sequence identification of mixed-sequence RNA/DNA oligonucleotides requires detailed understanding of the gas-phase dissociation of this class of compounds. The two major dissociation events, base loss and backbone fragmentation, are discussed and the unique fragmentation behavior of oligoribonucleotides is demonstrated. Backbone fragmentation of the all-RNA pentanucleotides is characterized by abundant c-ions and their complementary $y$-ions as the major sequence-defining fragment ion series. In contrast to the dissociation of oligodeoxyribonucleotides, where backbone fragmentation is initiated by the loss of a nucleobase which subsequently leads to the formation of the $\mathrm{w}$ - and [a-base]-ions, backbone dissociation of oligoribonucleotides is essentially decoupled from base loss. The different behavior of RNA and DNA oligonucleotides is related to the presence of the 2'-hydroxyl substituent, which is the only structural alteration between the DNA and RNA pentanucleotides studied. CID of mixed-sequence RNA/DNA pentanucleotides results in a combination of the nucleotide-typical backbone fragmentation products, with abundant $\mathrm{w}$-fragment ions generated by cleavage of the phosphodiester backbone adjacent to the deoxy building blocks, whereas backbone cleavage adjacent to ribonucleotides induces the formation of c- and y-ions. (J Am Soc Mass Spectrom 2002, 13, 936-945) (C) 2002 American Society for Mass Spectrometry
\end{abstract}

S ynthetic oligonucleotides play an increasing role in biomedical research as target compounds for pharmacological applications, as probes for cDNA hybridization or as triplex-forming oligonucleotides for recognition of specific DNA sequences [1]. Oligonucleotides of interest often exhibit unnatural nucleotide compositions, such as modified sugars and nucleobases, and thus, cannot be subjected to classical enzymatic sequencing techniques. Consequently, alternative sequencing techniques have to be evaluated and electrospray tandem mass spectrometry has the potential of becoming the method of choice for rapid and unambiguous sequence elucidation of synthetic oligonucleotides. Electrospray tandem mass spectrometry is one of the most versatile and accurate tools for structural investigation of biomolecules and it has successfully proven its potential in the field of peptide and protein analysis. The technique has found widespread applications in many laboratories for the determination of peptide sequence tags, identification of posttranslational modifications, and even for de novo sequencing of polypeptides. Furthermore, spectral interpretation is

Published online June 14, 2002

Address reprint requests to Dr. S. Schürch, Department of Chemistry and Biochemistry, University of Bern, Freiestrasse 3, CH-3012 Bern, Switzerland. E-mail: stefan.schuerch@ioc.unibe.ch supported by sophisticated software tools, which provide rapid protein identification and a fairly high sample throughput.

On the other hand, the elucidation of nucleotide sequences by tandem mass spectrometry lags far behind, mostly due to the highly complex fragment ion patterns generated by the dissociation of larger oligonucleotides. The dissociation of oligonucleotides is strongly influenced by the structural properties of the individual building blocks, such as the constitution of the nucleobases and the 2'-substituents, and many of the fundamental aspects of oligonucleotide dissociation are still unresolved. The majority of studies on the gas-phase dissociation of oligonucleotides has been focused on oligodeoxyribonucleotides (DNA) [2-5]. Based on the initial work of McLuckey et al. [6, 7], several research groups have further investigated the dissociation of DNA using either ESI or MALDI as the ionization method. As a result of these studies, a number of mechanisms explaining the loss of neutral or negatively charged nucleobases and the formation of the generally abundant [a-base]- and w-type fragment ions has been proposed. It is now widely accepted that the loss of the nucleobase, either by a 1,2-elimination process [8-14], by backside nucleophilic attack on the ribose $\mathrm{C} 1$ by the 3'-phosphate [15], or by the release of a negatively charged nucleobase and subsequent ab- 
straction of the nearby phosphate proton via a protonbound intermediate [16, 17], is the initial fragmentation step triggering the subsequent cleavage of the 3'-C-O bond. However, not all of the proposed mechanisms may account for the observed site-specificity of neutral and negative base loss. Recent studies by Gross and co-workers have greatly extended the knowledge of the processes involved in DNA backbone dissociation and the authors proposed a charge-remote fragmentation mechanism via a tripolar zwitterionic transition state [18-20]. Supporting data for this mechanism were further obtained from H/D-exchange experiments, which also provided information to reject the alternative 1,2elimination mechanism [21, 22]. However, from the present state of knowledge it cannot be excluded that, depending on the ion polarity and charge state, different mechanisms are involved in base loss and backbone dissociation of oligodeoxyribonucleotides.

Backbone fragmentation is a prerequisite for sequence elucidation of any biopolymer and the success of a tandem mass spectrometric sequencing procedure depends on its ability to generate oppositely directed series of sequence-defining fragment ions, such as the $\mathrm{w}$ - and [a-base]-series in the case of oligodeoxynucleotides. However, little is known about the dissociation of oligoribonucleotides and no information on the fragmentation behavior of mixed-sequence ribo-/ deoxyribo-(RNA/DNA) oligonucleotides is available yet. One of the first reports on the fragmentation of RNA has been given by Cerny et al. [15], who studied the metastable decomposition and the high-energy collision-induced dissociation of ribotrinucleotides in a magnetic sector instrument. They noticed a fragmentation pattern which is different from the one observed for deoxyribotrinucleotides and found characteristic fragment ions generated by cleavage across the 5'-P-O bond, which they referred to as the "Seq $\mathrm{xA}_{3}$ ' ions" ( $c_{x}$-ions). Ni et al. presented a computer-assisted strategy for sequence determination of oligonucleotides and applied it to the MS/MS spectra of short DNA-, RNA-, and modified oligonucleotide-sequences [23]. Though the algorithm is primarily based on the DNA-typical wand [a-B]-ions, assignment of RNA sequences was performed successfully. The loss of the nucleobase has been demonstrated to be the initial step in DNA fragmentation, whereas its role in the dissociation of RNA has not yet been elucidated completely. Due to the stabilizing effect of the 2'-hydroxyl substituent on the $\mathrm{N}$-glycosidic bond, base loss is expected to play a less predominant role in the fragmentation of RNA [24]. Griffey et al. described the gas-phase cleavage of a chimeric 27-mer oligonucleotide with deoxyadenosines incorporated in a 2'-O-methylribonucleotide sequence [25]. They demonstrated that fragmentation of these chimeric oligonucleotides is directed to the more labile deoxynucleotide sites, which finally results in abundant DNA-typical fragment ions. Recently, Kirpekar and Krogh presented data on the dissociation of singly protonated RNase digestion products in a MALDI-
QqTOF instrument [26]. They found the c- and y-ions as the most prevalent backbone dissociation products of RNA and emphasized the minor role base loss is playing upon backbone fragmentation of RNA.

We have investigated the fragmentation of a series of RNA and mixed-sequence pentanucleotides with deoxyribonucleotides selectively embedded within a sequence of ribonucleotides with the goal to gain insight into the mechanisms of base loss and backbone fragmentation.

\section{Experimental}

\section{Oligonucleotide Synthesis}

All oligonucleotides were synthesized in-house on a Amersham Pharmacia Biotech Gene-Assembler Special DNA synthesizer (Piscataway, NJ) using standard $\beta$-cyanoethyl phosphoramidite chemistry [27, 28]. Products were purified by ion pairing RP-HPLC, lyophilized, and redissolved in water prior to mass spectrometric analysis.

\section{Sample Preparation}

A mixture of acetonitrile/water/triethylamine (49+ $49+2$ ) was added to the aqueous oligonucleotide solution, resulting in a final oligonucleotide concentration of about $10 \mathrm{pmol} / \mu \mathrm{l}$. Typically, a sample volume of $2 \mu \mathrm{l}$ was used for analysis.

\section{Mass Spectrometry}

All experiments were performed on an Applied Biosystems/MDS Sciex QSTAR Pulsar hybrid quadrupoletime-of-flight mass spectrometer (Sciex, Concord, Canada), equipped with a nanoelectrospray ion source (Protana, Odense, Denmark). Oligonucleotides were analyzed in the negative-ion mode with a potential of $-850 \mathrm{~V}$ applied to the nanospray needle. Nitrogen was used as the curtain gas.

Tandem mass spectrometric experiments were performed with the precursor ions selected within a window of $\pm 1.5 \mathrm{~m} / \mathrm{z}$ units. Collision-induced dissociation was performed with collision energies in the range from 5 to $40 \mathrm{eV}$ using nitrogen as the collision gas. The time-of-flight analyzer was tuned for an average mass resolving power of 10,000 (FWHM) and calibrated externally using a mixture of caesium iodide and taurocholic acid. The Applied Biosystems Analyst QS software package was used for data processing.

\section{Results and Discussion}

A series of pentanucleotides based on the all-RNA pentamer UUUUU, with uridine selectively replaced by cytidine, deoxycytidine or deoxythymidine, was synthesized in order to investigate the basic fragmentation pattern of oligoribonucleotides and to study the influ- 
ence of deoxyribonucleotides embedded within a sequence of ribonucleotides. Pentanucleotides were chosen for this study since they can be dissociated efficiently under low-energy CID conditions and the sequences are large enough to study the positional selectivity of the dissociation processes. The major fragmentation events are the loss of either a neutral or a negatively charged nucleobase and the dissociation of the phosphodiester backbone, which results in the various series of analytically valuable sequence-defining ions.

\section{Neutral Base Loss}

The loss of either a negatively charged or a neutral nucleobase was observed as the primary low-energy dissociation event. Loss of neutral nucleobases was predominantly observed for the $[\mathrm{M}-2 \mathrm{H}]^{2-}$ precursor ions, whereas higher precursor ion charge states favor the loss of a negatively charged nucleobase. The product ion spectra of the doubly deprotonated all-RNA pentanucleotides CUUUU, UUUCU, and UUUUC revealed the positional selectivity of neutral base loss. The release of both types of neutral nucleobases, uracil and cytosine from CUUUU and UUUCU was observed, as indicated by the $[\mathrm{M}-2 \mathrm{H}-\mathrm{HB}(\mathrm{U})]^{2-}$ and $[\mathrm{M}-2 \mathrm{H}-$ $\mathrm{HB}(\mathrm{C})]^{2-}$ fragment ions with $\mathrm{m} / \mathrm{z} 676.62$ and $\mathrm{m} / \mathrm{z}$ 677.11, respectively (Figures 1a and $b$ ). The mass difference of $1 \mathrm{Da}$ between uridine and cytidine results in the ${ }^{13} \mathrm{C}$ isotopic peak of $[\mathrm{M}-2 \mathrm{H}-\mathrm{HB}(\mathrm{U})]^{2-}$ to overlap with the monoisotopic peak of $[\mathrm{M}-2 \mathrm{H}-\mathrm{HB}(\mathrm{C})]^{2-}$. In order to compare the behavior of the different pentanucleotide sequences, the contributions of the individual base losses to the total abundance of all base-loss fragments have been calculated and are summarized in Table 1. CUUUU and UUUCU both showed a preference for the loss of the neutral nucleobase from the cytidine, with the $[\mathrm{M}-2 \mathrm{H}-\mathrm{HB}(\mathrm{C})]^{2-}$ ion from CUUUU contributing $47 \%$ to the total of the base-loss fragments, though a single cytidine is present within each sequence only. Such a preference is most likely due to the enhanced proton affinity of the cytidine [29, 30], which facilitates the protonation of the nucleobase as the initial fragmentation step triggering the subsequent dissociation of the N-glycosidic bond. However, no evidence for the loss of a neutral cytosine from the 3 '-terminal cytidine of UUUUC (Figure 1c) was found, and despite the higher proton affinity of cytidine, uracil was released exclusively. The isotopic distribution of the corresponding $[\mathrm{M}-2 \mathrm{H}-\mathrm{HB}(\mathrm{U})]^{2-}$ ion with $\mathrm{m} / \mathrm{z}$ 676.61 was found to closely correlate with the theoretical isotopic distribution and any significant contribution from an overlapping $[\mathrm{M}-2 \mathrm{H}-\mathrm{HB}(\mathrm{C})]^{2-}$ fragment ion with a monoisotopic $\mathrm{m} / \mathrm{z}$ of 677.11 could be excluded.

In order to study the influence of deoxyribonucleotides on the dissociation, a set of mixed-sequence pentanucleotides with deoxycytidine and deoxythymidine embedded within a sequence of ribonucleotides was
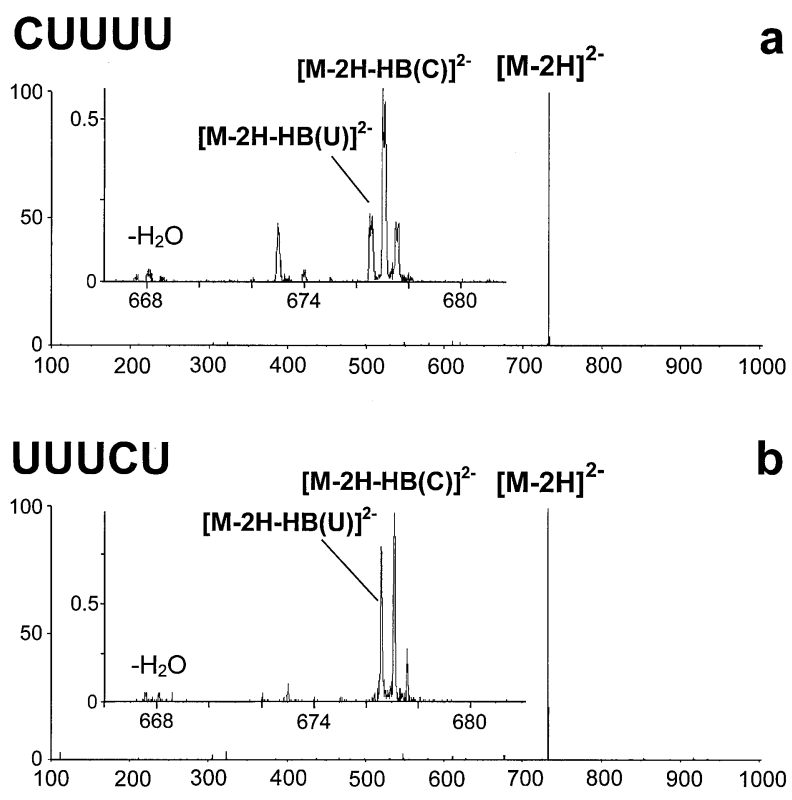

\section{UUUUC}

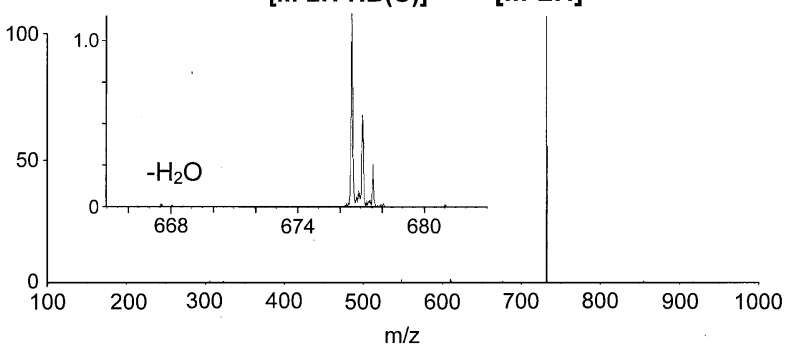

Figure 1. Product ion spectra of the doubly deprotonated allRNA pentanucleotides CUUUU (a), UUUCU (b), and UUUUC (c), recorded at the onset of dissociation with a collision energy of 10 $\mathrm{eV}$. The spectra demonstrate the loss of neutral nucleobases as the primary low-energy dissociation events which may be followed by the loss of water.

subjected to CID. Considering the lack of a stabilizing 2'-hydroxyl substituent, an increased trend for base loss from the deoxynucleotide was expected [24]. Substituting the cytidines in CUUUU and UUUCU for deoxycytidines resulted in an increase of cytosine-loss (Table 1). However, no preference for the loss of the nucleobase from the deoxythymidines in UUdTdTC and UUUdTC was observed. The loss of the nucleobase from uridine was still highly preferred (79-93\%), whereas neutral base loss from the deoxythymidine was about an order of magnitude less prevalent.

\section{Neutral Base Loss from the 3'-Terminal Nucleotide}

The influence of the deoxynucleotide on neutral base loss became apparent when the 3'-terminal cytidine in UUUUC was replaced with a deoxycytidine. In contrast to the all-ribo-pentanucleotide, which clearly disfavors the loss of the 3'-terminal nucleobase, the loss of cytosine from UUUUdC is a significant low-energy dissoci- 
Table 1. Preferences for neutral and negative nucleobase loss from doubly and triply deprotonated pentanucleotides given as percentages of the individual base loss fragments relative to the total peak abundance of all base loss fragments

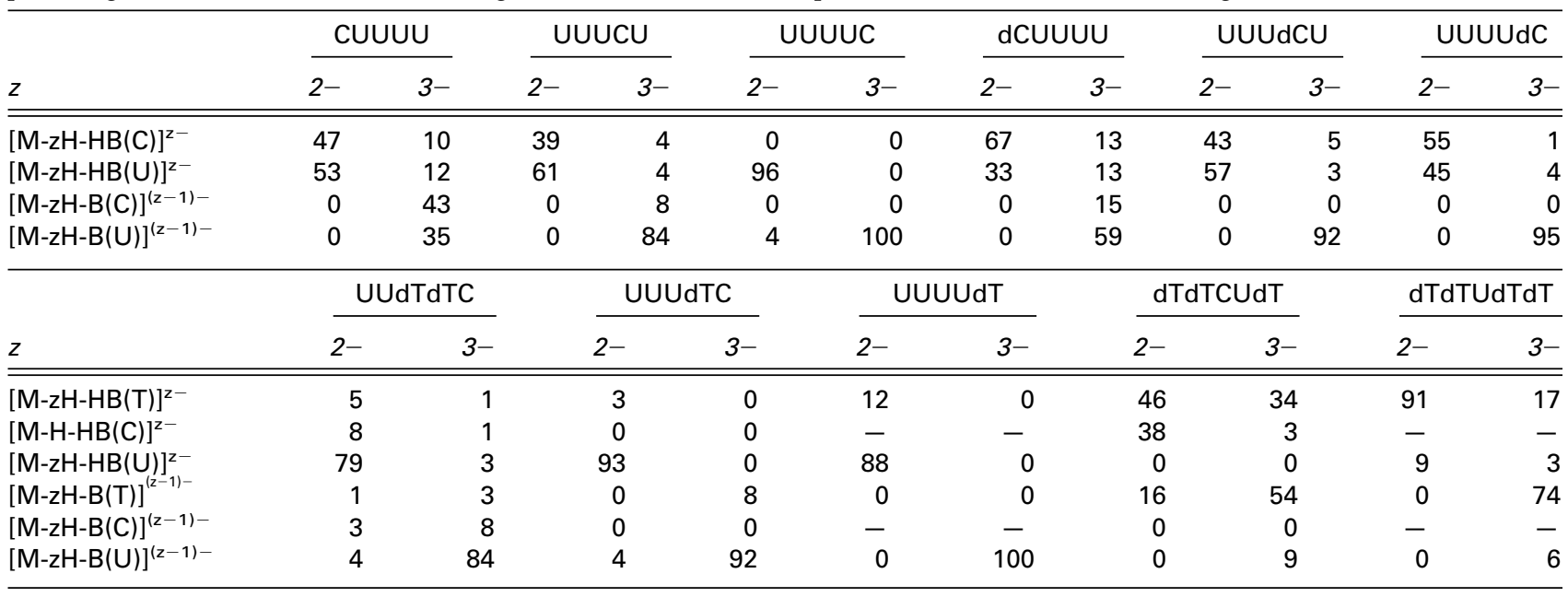

Data obtained from $10 \mathrm{eV}$ product ion spectra.

$z$ indicates the precursor ion charge state.

$[\mathrm{M}-\mathrm{zH}-\mathrm{HB}(\mathrm{X})]^{\mathrm{z}-}$ are ions generated by neutral base loss.

$[\mathrm{M}-\mathrm{zH}-\mathrm{B}(\mathrm{X})]^{(\mathrm{z}-1)-}$ are ions generated by negative base loss.

ation event, as reflected by the peak of the $[\mathrm{M}-2 \mathrm{H}-$ $\mathrm{HB}(\mathrm{C})]^{2-}$ fragment ion with $\mathrm{m} / \mathrm{z} 669.10$ in Figure 2a. If the increased trend for loss of the neutral nucleobase from the 3'-terminal nucleotide were solely attributed to the absence of the 2'-hydroxyl substituent and the hence reduced stability of the N-glycosidic bond of the deoxyribonucleotide, an identical behavior would be expected for UUUUdT. However, CID experiments showed no extensive loss of thymine and the release of uracil was still highly preferred with a contribution of $88 \%$ to the total abundance of all base-loss fragments (see Table 1 and Figure $2 b$ ). Furthermore, fragment ions with $\mathrm{m} / \mathrm{z} 660.08$, indicating the loss of water from [M $2 \mathrm{H}-\mathrm{HB}(\mathrm{C})]^{2-}$ and $[\mathrm{M}-2 \mathrm{H}-\mathrm{HB}(\mathrm{T})]^{2-}$, were found in the product ion spectra of UUUUdC and UUUUdT, respectively. Up to $50 \%$ of the primary base-loss fragments further decomposed within the time scale of the CID experiment by the release of a water molecule, whereas the fragments of pentanucleotides with a 3'terminal ribonucleotide were virtually immune to such decomposition (see Figure 1).

\section{Neutral Base Loss from Pentamers with a Deoxyribose Template}

For comparison, the dissociation of two pentanucleotides with high deoxyribonucleotide contents was examined. $d T d T C U d T$ and $d T d T U d T d T$ both showed a great preference for the loss of the nucleobase from deoxythymidine (up to $91 \%$ ), and the release of uracil was much less prevalent than for the pentanucleotides studied previously. The origin of the released nucleobase was not directly identified by the MS/MS experiments. Nevertheless, the low abundance of the thymine-loss fragments from UUdTdTC $(5 \%)$, UUUdTC $(3 \%)$, and UUUUdT (12\%), and the highly increased loss of thy- mine from dTdTCUdT (46\%) and dTdTUdTdT (91\%) point to the 5'-terminal deoxythymidine as the most probable site of neutral base loss. The competition between the release of the nucleobase from the positionally preferred 5'-terminal nucleotide and the alternative loss of the nucleobase with the highest proton affinity is reflected by the product ion spectrum of $d T d T C U d T$, which showed neutral base loss from the single cytidine to be almost as prevalent as from the deoxythymidines (Table 1). On the other hand, neutral base loss from the low proton affinity uridine in dTdTCUdT was not observed and was an insignificant dissociation event for dTdTUdTdT as well.

The following conclusions can be drawn from the results obtained so far: (1) Neutral base loss exhibits a positional selectivity with a great preference for the release of the 5'-terminal nucleobase, whereas the loss of the nucleobase from the 3'-terminal nucleotide is strongly hindered. (2) Nucleobases with higher proton affinity $(\mathrm{C} \gg \mathrm{T}>\mathrm{U})$ are predominantly released. (3) The 2'-substituent does not significantly affect the preferences for neutral base loss. (4) Loss of the 3'-terminal nucleobase becomes a significant dissociation event in the case of a deoxyribonucleotide with high proton affinity (e.g., loss of cytosine from UUUUdC).

The observed preference for neutral base loss from low charge state precursors agrees well with the mechanism based on the formation of a zwitterionic intermediate by proton transfer from an adjoining phosphate group $[18,19,31]$. This mechanism favors the loss of a neutral nucleobase as long as proton transfer is feasible. Higher precursor ion charge states diminish the availability of acidic phosphate protons, thus lowering the probability of zwitterion formation and giving rise to the alternative loss of a negatively charged nucleobase. The base specificity of neutral base loss is reflected by 


\section{UUUUdC}

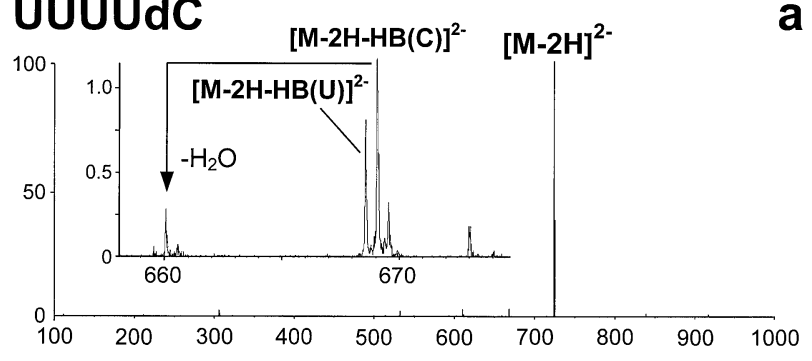

\section{UUUUdT}

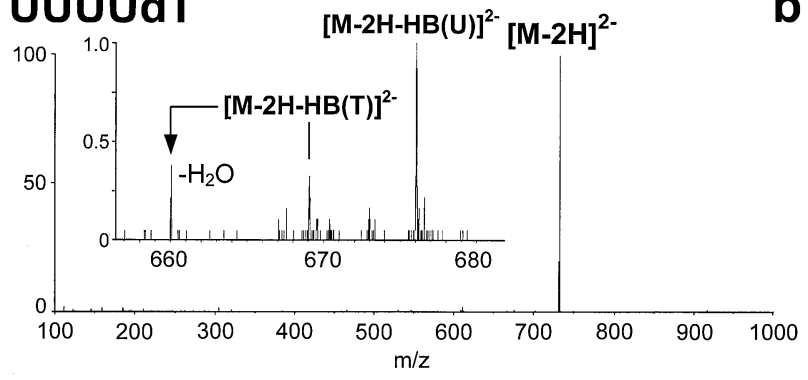

Figure 2. Product ion spectra $(10 \mathrm{eV})$ of the doubly deprotonated pentanucleotides UUUUdC (a) and UUUUdT (b). The loss of the 3'-terminal cytosine from UUUUdC is the preferred lowest-energy dissociation pathway, whereas the loss of the neutral thymine from UUUUdT is less prevalent. Both base losses are followed by the loss of a water molecule.

the relatively high abundance of the $[\mathrm{M}-2 \mathrm{H}-$ $\mathrm{HB}(\mathrm{C})]^{2-}$ fragment ions in the spectra of pentanucleotides containing nucleotides with increased proton affinity, such as cytidine or deoxycytidine, which facilitate the protonation of the nucleobase. The presence of a deoxynucleotide within a ribonucleotide sequence had an unexpected small influence on neutral base loss. Despite the decreased gas-phase stability of the Nglycosidic bond in DNA [24], the product ion spectra of dCUUUU, UUUdCU, UUdTdTC, and UUUdTC gave no evidence for a significantly increased base loss from the deoxyribonucleotides, and neutral base loss basically followed the same preferences as for the all-RNA pentanucleotides. Thus, the preferences for neutral base loss must essentially be given by the ease of zwitterion formation as the mechanistic key event, and within this context, the slightly weakened N-glycosidic bond of the deoxyribonucleotides has no significant influence on the overall process. The water loss observed in conjunction with the loss of the 3'-terminal nucleobase from UUUUdC and UUUUdT reflects the DNA-typical backbone fragmentation with the protonated 3'-OH group in place of the phosphate as the leaving group. Consequently, a water molecule will be released as a $w_{0}$ fragment.

The proton transferred to the nucleobase was originally proposed to originate from the adjoining 5'phosphate group [18]. This sterically favored process, however, cannot explain the protonation and subsequent release of the 5'-terminal nucleobase. Wan et al. [22] recently showed the loss of the 5'-terminal guanine nucleobase from $d(G T T T)$, demonstrating that other sites than the adjacent 5'-phosphate, e.g., the 3'-phosphate or a remote phosphate group, might serve as proton sources, and such an alternative protonation mechanism might also explain the loss of cytosine from CUUUU and dCUUUU. The facts that the loss of a neutral cytosine from $d C U U U U$ was even more prevalent than from UUUUdC, and that the pentanucleotides with a 5'-terminal deoxythymidine (dTdTCUdT and dTdTUdTdT) showed an increased loss of thymine, implies that protonation of the nucleobase by the 3'phosphate is an important alternative mechanism.

Several of the nucleotide sequences were designed to study the positional selectivity of neutral base loss. These sequences exhibit a unique terminal nucleobase, which allowed to unambiguously identify the origin of the released nucleobase. The positional selectivity of neutral base loss was strong for sequences with a terminal ribonucleotide. Loss of the 3'-terminal nucleobase from UUUUC, UUdTdTC, and UUUdTC was hardly observed (Table 1). On the other hand, the loss of cytosine from the 3'-terminal deoxycytidine of UUUUdC was found to be the main lowest-energy dissociation event.

\section{Loss of Negatively Charged Bases}

The loss of a negatively charged nucleobase is the predominant low-energy dissociation event of triply and quadruply charged pentanucleotides. The loss of a negatively charged base is indicated by a low-mass fragment ion characterizing the type of nucleobase expelled ( $m / z 110.02$ for cytosine, $m / z 111.02$ for uracil, and $m / z 125.03$ for thymine) and a complementary [M $\left.\mathrm{nH}-\mathrm{B}^{-}\right]^{(\mathrm{n}-1)-}$ ion of reduced charge state. The relative abundances of fragment ions generated by the loss of negatively charged nucleobases are also summarized in Table 1. Though these fragments are of the most abundant low-energy dissociation products of the triply charged precursors, they are not generated exclusively and the occurrence of secondary fragmentation processes, which remove primary fragment ions from the spectrum, cannot be excluded. This is indicated by the negatively charged cytosine and uracil ions, which are often more abundant than their $\left[\mathrm{M}-3 \mathrm{H}-\mathrm{B}^{-}\right]^{2-}$ counterparts.

The positional selectivity of charged base loss from triply charged precursor ions is reflected by the relative fractions of the $\left[\mathrm{M}-3 \mathrm{H}-\mathrm{B}^{-}\right]^{2-}$ ion intensities summarized in Table 1. Data indicate that the loss of a negatively charged base from the 5'-terminal ribonucleotide is generally favored and that the loss of the 3'-terminal base is hardly observed. The $[\mathrm{M}-3 \mathrm{H}-$ $\left.\mathrm{B}_{1}^{-}\right]^{2-}$ ions are typically an order of magnitude more abundant than the fragments generated by the loss of any other negatively charged base. The data for UUdTdTC, UUUdTC, and UUUUdT in the lower section of Table 1 show an incremental increase of the $[\mathrm{M}-3 \mathrm{H}$ $-\mathrm{B}(\mathrm{U})]^{2-}$ ions from 84 to $100 \%$. Assuming that all the 

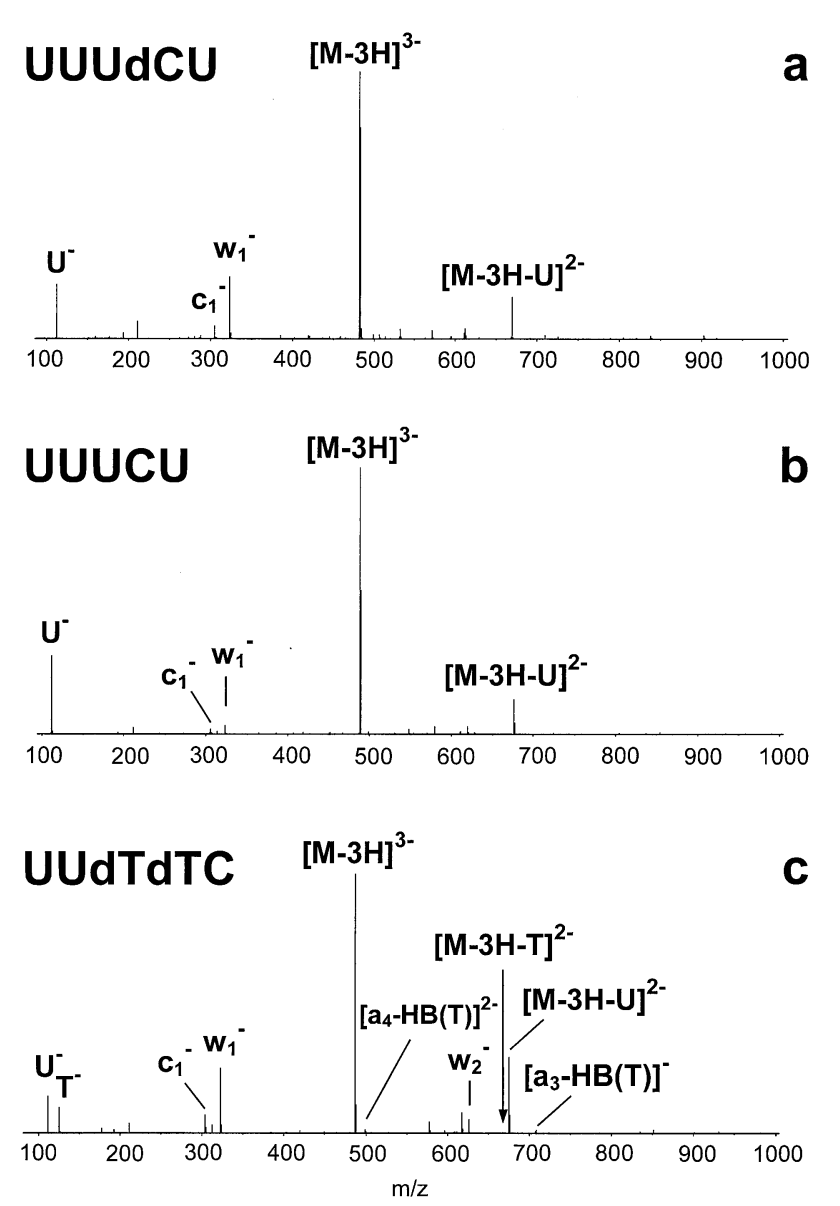

Figure 3. Product ion spectra of the triply deprotonated pentanucleotides UUUdCU (a), UUUCU (b), and UUdTdTC (c), showing the loss of the negatively charged nucleobases as the primary low-energy dissociation events. Base loss from the deoxyribonucleotides in UUUdCU and UUdTdTC are immediately followed by the DNA-typical cleavage of the 3'-C-O bond, as reflected by the presence of abundant $\mathrm{w}$-type ions. $\mathrm{U}^{-}$and $\mathrm{T}^{-}$refer to the negatively charged nucleobases uracil and thymine. Spectra recorded with threshold collision energy of $10 \mathrm{eV}$.

non-terminal uridines are equivalent, they contribute less than $10 \%$ each to the total peak abundance and the main fraction must originate from the loss of the 5'-terminal uracil. Similar conclusions can be drawn from comparison of the data in the upper section of Table 1, which show a significant loss of negatively charged cytosine only if cytidine or deoxycytidine is located at the 5'-terminus of the pentanucleotide.

Loss of a negatively charged nucleobase from triply charged precursor ions is demonstrated by the product ion spectra of UUUdCU, UUUCU, and UUdTdTC in Figure 3, which have been recorded with a collision energy of $10 \mathrm{eV}$. The spectra show the $[\mathrm{M}-3 \mathrm{H}-$ $\mathrm{B}(\mathrm{U})]^{2-}$ ion as the most characteristic low-energy dissociation product, whereas the released uracil anion is characterized by the peak at $m / z 111.02$. The product ion spectrum of triply charged UUdTdTC, represented by Figure $3 c$, shows the uracil and thymine ions with $\mathrm{m} / \mathrm{z}$ 111.02 and $m / z 125.04$, respectively. The complementary $[\mathrm{M}-3 \mathrm{H}-\mathrm{B}(\mathrm{U})]^{2-}$ ion is reflected by the abundant peak at $m / z 674.64$, whereas the $[\mathrm{M}-3 \mathrm{H}-\mathrm{B}(\mathrm{T})]^{2-}$ fragment ion with $\mathrm{m} / \mathrm{z} 667.12$ is of extremely low abundance, implying that the primary base-loss fragment readily decomposes. Furthermore, abundant wtype fragment ions originating from backbone dissociation adjacent to the deoxyribonucleotides appear in the spectra of UUUdCU and UUdTdTC.

The results summarized in Table 1 demonstrate that the loss of the 3'-terminal nucleobase from triply charged precursor ions is strongly hindered. No loss of a negatively charged cytosine from UUUUC, UUUUdC, UUUdTC, or thymine from UUUUdT was observed. Charged base loss from the 5'-terminal nucleotide was clearly the favored dissociation event and sequences with a 5'-terminal uridine or deoxythymidine exhibited an almost exclusive loss of the 5'-terminal nucleobase. On the other hand, the loss of the charged 5'-terminal nucleobase from CUUUU and prevalent as for the other pentanucleotides investigated and a significant contribution from the loss of the uracil anion was still observed. Among the nucleotides studied, cytidine exhibits the lowest preference to release its nucleobase as an anion, which is consistent with the general order of $\mathrm{A}^{-}<\mathrm{T}^{-}<\mathrm{G}^{-}, \mathrm{C}^{-}$observed for charged base loss from multiply charged oligodeoxyribonucleotides $[8,9]$. So far, the data obtained by CID of pentanucleotides do not indicate any correlation between the preferences for charged base loss and the 2 '-substituent. The corresponding pairs of pentanucleotides with cytidine in place of the deoxycytidine (CUUUU/dCUUUU, UUUСU/UUUаCU, and UUUUС/ UUUUdC) exhibit quite similar behavior. The decreased abundance of the $[\mathrm{M}-3 \mathrm{H}-\mathrm{B}(\mathrm{C})]^{2-}$ ion in the spectrum of $d C U U U U$ (compared to CUUUU) may not be indicative for the absolute degree of charged base loss from the 5'-terminal deoxycytidine, since it may be related to further decomposition of the primary dissociation product.

\section{Backbone Fragmentation of all-RNA Pentanucleotides}

CID of the doubly charged UUUUU pentamer yielded multiple series of backbone fragmentation products with a mass difference of 306.03 Da between adjacent peaks reflecting the nucleotide sequence. However, assignment of the fragment ion series was ambiguous, since the oppositely directed $a / z, b / y, c / x$, and $d / w$ ion series of the uridine pentamer all are isobaric. Replacing one of the uridines $(306.03 \mathrm{Da})$ with a cytidine $(305.04 \mathrm{Da})$ introduces a mass difference of $1 \mathrm{Da}$ between the 5'- and 3'-terminal product ions, which allows the fragment ion series to be assigned unambiguously. The product ion spectrum of UUUUC (Figure 4a) reveals the c-ions $\left(\mathrm{c}_{1}^{-}, \mathrm{m} / \mathrm{z} 305.02 ; \mathrm{c}_{2}^{-}, \mathrm{m} / \mathrm{z} 611.04 ; \mathrm{c}_{3}^{-}\right.$, $\mathrm{m} / \mathrm{z}$ 917.07) and their complementary y-ions $\left(\mathrm{y}_{2}^{-}, \mathrm{m} / \mathrm{z}\right.$ $\left.548.10 ; \mathrm{y}_{3}^{-}, \mathrm{m} / \mathrm{z} 855.13 ; \mathrm{y}_{4}^{-}, \mathrm{m} / \mathrm{z} 1161.15\right)$ as the main backbone dissociation products. The fragment ions are 

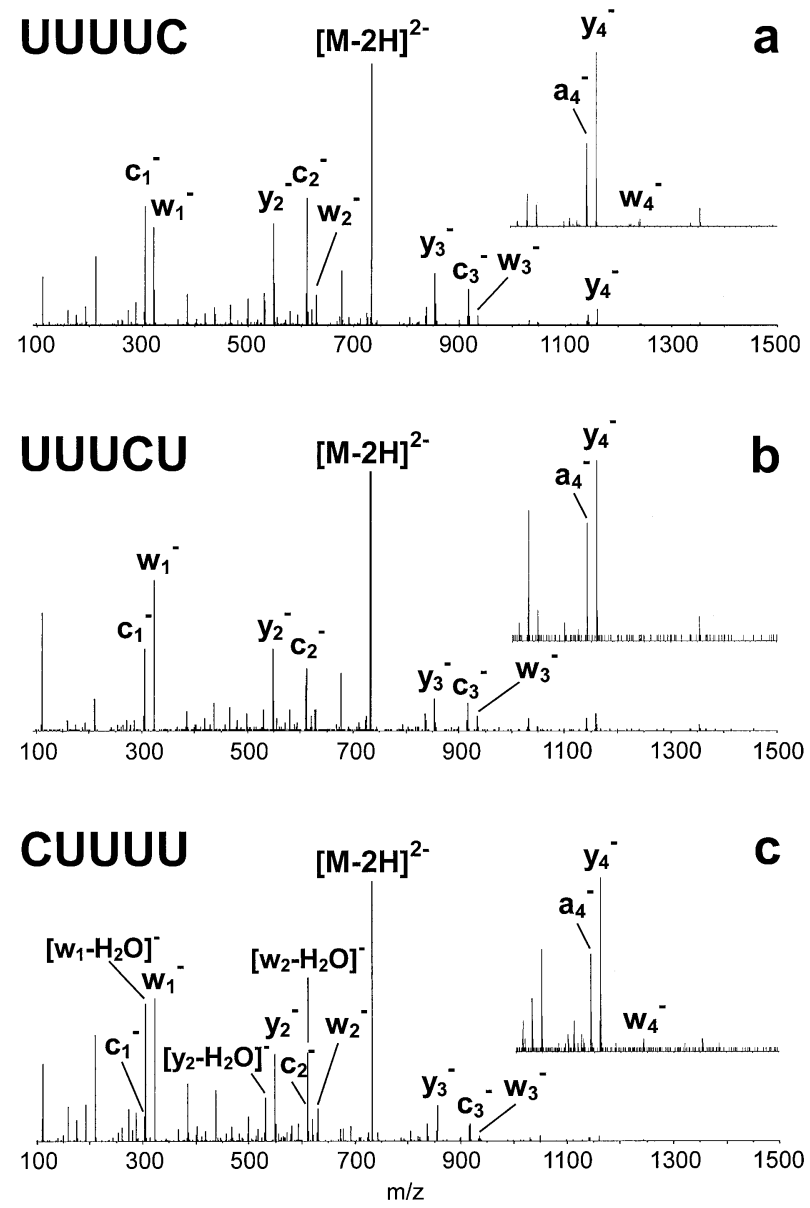

Figure 4. Product ion spectra of the all-RNA pentanucleotides UUUUC (a), UUUCU (b), and CUUUU (c) obtained by dissociation of the $[\mathrm{M}-2 \mathrm{H}]^{2-}$ precursor ions with a collision energy of 30 $\mathrm{eV}$. The main sequence-defining fragment ions are the $\mathrm{c}-, \mathrm{y}-$ and w-series.

labeled according to the nomenclature proposed by McLuckey et al. [6]. The $y_{1}^{-}$ion was not detected due to the lack of a suitable deprotonation site, but the missing 3 'terminal sequence information was readily obtained from the $\mathrm{w}$-ion series $\left(\mathrm{w}_{1}^{-}, \mathrm{m} / \mathrm{z} 322.04 ; \mathrm{w}_{2}^{-}, \mathrm{m} / \mathrm{z}\right.$ 628.07; $\mathrm{w}_{3}^{-}, m / z$ 934.09; and $\mathrm{w}_{4}^{-}, m / z$ 1240.12). These DNAtypical $\mathrm{w}$-type fragment ions were detected with an unexpected high abundance in the product ion spectra of the all-RNA pentanucleotides. However, as noted by Wang et al. [18], who observed a generally higher abundance of the w-ions than of their complementary [a-base]-ions in the product ion spectra of oligodeoxyribonucleotides, there may exist alternative ways to the proposed DNA-typical dissociation mechanism for the generation of $\mathrm{w}$-ions. An alternative mechanism for the formation of $\mathrm{w}$-ions in the absence of nucleobase loss, involving the abstraction of the 4'-proton by the 3'phosphate group, was recently proposed by Wan et al. [22]. A similar mechanism was presented by Bartlett et al. for the backbone cleavage of methylphosphonateoligodeoxynucleotides [32], and such a mechanism might also apply to the dissociation of the RNA-oligo- nucleotides. The types of fragment ions generated by CID of the pentanucleotides were essentially independent of the collision energy and the product ion distribution was reproducible as long as the energy was not excessively high. Basically, most of the theoretically possible backbone fragmentation products were detected upon CID and supporting sequence information was obtained from the multiple ion series. However, the multitude of fragment ions greatly increases the complexity of the spectra and may lead to ambiguous peak assignments. Spectral interpretation is further complicated by the presence of nucleobase losses in conjunction with backbone dissociation, which was primarily observed for the a-ions, and to a lesser degree also for the other ion series. The enhanced preference for the a-ions to be generated in conjunction with neutral base loss is reflected by the ratios of the a- and $[\mathrm{a}-\mathrm{HB}]$ peak abundances, with an $\mathrm{a} /[\mathrm{a}-\mathrm{HB}]$ ratio on the order of 1.0 demonstrating that both types of fragment ions are formed to the same degree. For the other ion series, higher ratios in the range from 1.6 to 5.5 indicate a decreased trend for neutral base loss in conjunction with backbone fragmentation. Furthermore, the number of fragment ions may be increased by an additional loss of water from the primary fragments. Nevertheless, the information provided by the abundant $\mathrm{c}^{-}, \mathrm{y}-$ and $\mathrm{w}$-ion series is sufficient for unambiguous identification of isomeric nucleotide sequences, as demonstrated by the spectra of UUUUC, UUUCU, and CUUUU in Figure 4.

\section{Backbone Fragmentation of Mixed-Sequence RNA/ DNA Pentanucleotides}

Considering the fragmentation of oligodeoxyribonucleotides, the generation of abundant $\mathrm{w}$ - and [a-HB]-ions due to cleavage of the $3^{\prime}-\mathrm{C}-\mathrm{O}$ phosphate bond adjacent to the dexoyribonucleotide was also expected for the mixed-sequence pentanucleotides. In order to verify the preferred dissociation pathways of mixed-sequence RNA/DNA-pentanucleotides, the relative abundances of the competing $\mathrm{w} / \mathrm{y}$ and $[\mathrm{a}-\mathrm{HB}] / \mathrm{c}$ ion series were compared and the corresponding data are summarized in Table 2. Most illustrative are the data for UUUdTC and UUdTdTC, and as demonstrated by the spectra in Figures $5 a$ and $b$, the DNA-typical $w$-ions became the most abundant dissociation products. The presence of the deoxythymidine in UUUdTC is indicated by the $\mathrm{w}_{1}^{-}$ ion with $\mathrm{m} / \mathrm{z} 322.05$, which is the base peak in the spectrum. For UUdTdTC, the presence of the two deoxythymidines is reflected by the simultaneous occurrence of the very abundant $\mathrm{w}_{1}^{-}$and $\mathrm{w}_{2}^{-}$fragment ions with $\mathrm{m} / \mathrm{z} 322.05$ and $\mathrm{m} / \mathrm{z}$ 626.10, respectively. Simultaneously, the formation of the competing (RNA-typical) $\mathrm{y}_{2}^{-}$ion is significantly reduced, resulting in a $\mathrm{w}_{2}^{-} / \mathrm{y}_{2}^{-}$ ratio of 12:1. No such calculation was possible for the $\mathrm{w}_{1}^{-} / \mathrm{y}_{1}^{-}$ion pair, since the $\mathrm{y}_{1}^{-}$ion was never detected. Similarly, backbone cleavage on the 3'-side of a deoxyribonucleotide resulted in an increased abundance of 
Table 2. Ratios of the ion abundances of the competing DNA- and RNA-typical fragment ions obtained from $30 \mathrm{eV}$ product ion spectra

\begin{tabular}{|c|c|c|c|c|c|c|c|c|c|c|c|c|c|}
\hline \multirow[b]{2}{*}{$z$} & \multicolumn{2}{|c|}{ CUUUU } & \multicolumn{2}{|c|}{ UUUCU } & \multicolumn{2}{|c|}{ UUUUC } & \multicolumn{3}{|c|}{ dCUUUU } & \multicolumn{2}{|c|}{ UUUdCU } & \multicolumn{2}{|c|}{ UUUUdC } \\
\hline & $2-$ & $3-$ & $2-$ & $3-$ & $2-$ & $3-$ & $2-$ & & $3-$ & $2-$ & $3-$ & $2-$ & $3-$ \\
\hline$\left[\mathrm{a}_{2}-\mathrm{HB}\right] / \mathrm{c}_{2}$ & 0.4 & 1.4 & 0.2 & 0.7 & 0.1 & 0.5 & 0.3 & & 0.8 & 0.1 & 0.6 & 0.1 & 0.7 \\
\hline$\left[\mathrm{a}_{3}-\mathrm{HB}\right] / \mathrm{c}_{3}$ & 0.4 & 2.5 & 0.3 & 1.1 & 0.2 & 0.7 & 0.1 & & 0.8 & $(n / a)^{b}$ & 1.1 & $(n / a)^{b}$ & 0.9 \\
\hline$\left[\mathrm{a}_{4}-\mathrm{HB}\right] / \mathrm{c}_{4}$ & 0.4 & 2.1 & 0.5 & 0.4 & 0.4 & 0.5 & 0.3 & & 0.7 & 5.2 & 8.8 & 0.2 & 0.5 \\
\hline $\mathrm{w}_{2} / \mathrm{y}_{2}$ & 0.4 & 2.4 & 0.3 & 0.8 & 0.3 & 0.7 & 0.5 & & 0.9 & 0.3 & 0.7 & 0.2 & 0.9 \\
\hline$w_{3} / y_{3}$ & 0.2 & 0.9 & 0.8 & 1.2 & 0.3 & 0.8 & 0.3 & & 0.1 & 0.2 & 0.7 & 0.3 & 0.8 \\
\hline \multirow[t]{2}{*}{$\mathrm{w}_{4} / \mathrm{y}_{4}$} & 1.3 & 1.2 & 0.5 & 1.1 & 0.5 & 1.0 & 9.3 & & 3.3 & 0.4 & 0.8 & 0.9 & 1.0 \\
\hline & \multicolumn{3}{|c|}{ UUdTdTC } & \multicolumn{2}{|c|}{ UUUdTC } & \multicolumn{3}{|c|}{ UUUUdT } & & \multicolumn{2}{|c|}{ dTdTCUdT } & \multicolumn{2}{|c|}{ dTdTUdTdT } \\
\hline$z$ & $2-$ & & $3-$ & $2-$ & $3-$ & $2-$ & & $3-$ & & $2-$ & $3-$ & $2-$ & $3-$ \\
\hline$\left[\mathrm{a}_{2}-\mathrm{HB}\right] / \mathrm{c}_{2}$ & 0.4 & & 1.5 & 0.4 & 0.7 & 0.2 & & 0.6 & & 3.4 & 14.4 & 1.0 & 1.1 \\
\hline$\left[\mathrm{a}_{3}-\mathrm{HB}\right] / \mathrm{c}_{3}$ & 4.7 & & 10.0 & 0.3 & 1.0 & 0.4 & & 0.8 & & 0.3 & 1.3 & 0.1 & 0.4 \\
\hline$\left[\mathrm{a}_{4}-\mathrm{HB}\right] / \mathrm{c}_{4}$ & 3.0 & & 2.9 & 2.5 & 3.2 & 0.9 & & 1.1 & & 0.2 & 0.3 & 4.4 & 1.3 \\
\hline $\mathrm{w}_{2} / \mathrm{y}_{2}$ & 12.0 & & 14.6 & 0.4 & 0.9 & 0.4 & & 0.8 & & 0.9 & 1.0 & 0.5 & 2.3 \\
\hline$w_{3} / y_{3}$ & 0.4 & & 0.5 & 0.3 & 0.9 & 0.2 & & 0.8 & & 2.9 & 2.6 & 0.8 & 2.2 \\
\hline$w_{4} / y_{4}$ & 0.6 & & 1.2 & 0.6 & 1.3 & 0.5 & & 1.0 & & 5.4 & 3.9 & 7.8 & 9.5 \\
\hline
\end{tabular}

[a-HB]- and w-ions are DNA-typical, c- and y-ions are RNA-typical. $z$ indicates the precursor ion charge state.

$n / a$ indicates ratios that could not be calculated due to overlapping fragment ion peaks.

Backbone fragmentation on the 3'-side of a deoxynucleotide is reflected by high [a-HB]/c and w/y ratios.

the $[\mathrm{a}-\mathrm{HB}]$ ion and a simultaneously decreased abundance of the c-ion, which is reflected by a correspondingly high $[\mathrm{a}-\mathrm{HB}] / \mathrm{c}$ ratio. For example, the $\left[\mathrm{a}_{4}-\mathrm{HB}\right] / \mathrm{c}_{4}$ ratio of UUUdCU showed a more than tenfold increase over UUUCU. The absence of a 3'-terminal phosphate group impedes the formation of a DNA-typical fragment ion from pentanucleotides with a 3'-terminal deoxyribonucleotide. Thus, the product ion spectrum of UUUUAC (Figure 5c) exhibits the same types of fragment ions as the spectrum of the corresponding allRNA oligonucleotide UUUUC (Figure 4a). The [a-HB]/ $\mathrm{C}$ and $\mathrm{w} / \mathrm{y}$ ratios calculated for $d T d T U d T d T$ are not characteristic for the distribution of the DNA and RNA building blocks within the sequence, since the symmetric nucleotide sequence causes the $c / x$ and the $b / y$ ions to be isobaric and consequently, the contributions of the individual c- and y-ions to the peak abundances cannot be determined. Similar results were obtained by dissociation of triply charged precursor ions, as demonstrated by the $\mathrm{w}_{1}$ and $\mathrm{w}_{2}$ fragment ions in the product ion spectra of UUUdCU and UUdTdTC in Figure 3.

Data obtained from CID of selected pentanucleotides provide insight into the mechanisms of RNA-backbone fragmentation. Observations indicate that the fragmentation behavior of oligoribonucleotides differs significantly from the dissociation of oligodeoxyribonucleotides, which has to be related to the presence of the 2'-hydroxyl group, since this is the only structural variation between DNA and RNA. RNA-specific dissociation is reflected by the formation of $\mathrm{c}$ - and $\mathrm{y}$-ions as the major backbone fragmentation products and by the fact that this process is essentially decoupled from base loss.

A simple mechanism with the acidic phosphate proton transferred to the nearby 5'-oxygen and subse- quent cleavage of the 5'-P-O bond might be applicable to the backbone fragmentation of RNA (Scheme 1a). However, such a mechanism does not account for the influence of the 2'-ribose substituent and gives no explanation why DNA and RNA do exhibit a generally different fragmentation behavior. Given the fact that RNA differs from DNA by the additional 2'-hydroxyl group, the 2'-substituent must be the key element involved in the dissociation mechanism. A potential mechanism for oligoribonucleotide dissociation is depicted in Scheme 1b. Backbone dissociation is initiated by the formation of an intramolecular cyclic transition state with the 2'-hydroxyl proton bridged to the 5'phosphate oxygen. Following abstraction of the 2'hydroxyl proton, the 5'-P-O bond dissociates and releases the c- and y-type fragments. Subsequent bond rearrangement leads to the formation of a 3'-metaphosphoric-acid ester group, which stabilizes the c-type fragment. The $\mathrm{y}_{1}$-type fragment released as a result of backbone cleavage adjacent to the 3'-terminal nucleotide is a neutral and consequently, will not be detected. In the presence of an adjacent neutral phosphate group, dissociation will take place in conjunction with the translocation of the phosphate proton to the negatively charged 2'-oxygen, resulting in an uncharged 3'-terminus. Such a mechanism might also account for the formation of $\mathrm{c}^{-}$and $\mathrm{y}$-type ions as a result of the dissociation of singly protonated MALDI-generated oligoribonucleotides [26].

\section{Conclusions}

This work demonstrates the unique dissociation behavior of oligoribonucleotides (RNA) upon low-energy 

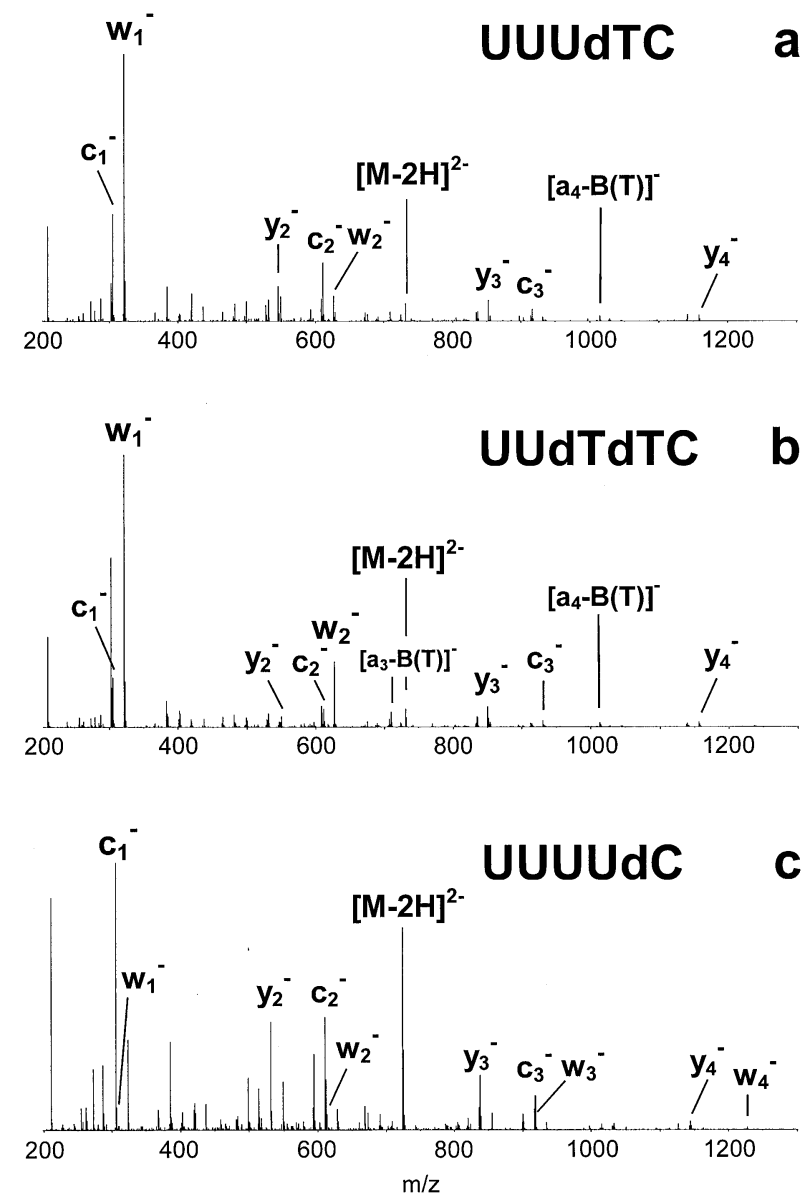

Figure 5. Product ion spectra of the mixed-sequence RNA/DNA pentanucleotides UUUdTC (a), UUdTdTC (b), and UUUUdC (c). Dissociation of doubly charged precursor ions with a collision energy of $30 \mathrm{eV}$. The presence of a deoxyribonucleotide within the RNA-sequence is indicated by abundant $\mathrm{w}_{1^{-}}$and $\mathrm{w}_{2}$-fragment ions. Dissociation of the UUUUdC pentanucleotide with a 3'terminal deoxycytidine does not generate an indicative w-type fragment ion.

CID. In contrast to the dissociation of oligodeoxyribonucleotides (DNA), which has been investigated thoroughly within the last few years, most of the mechanistic aspects of RNA-dissociation are still unresolved. Unlike oligodeoxyribonucleotides, which predominantly decompose into the [a-base]- and $\mathrm{w}$-ions, the product ion spectra of oligoribonucleotides are characterized by the abundant peaks of the c- and their complementary y-ions. Furthermore, backbone fragmentation of RNA is decoupled from base loss. The corresponding fragmentation mechanism involves the 2'-hydroxyl substituent as the structural key element, thus accounting for the altered behavior of RNA. Investigation of mixed-sequence RNA/DNA-oligonucleotides demonstrates that the characteristic fragmentation mechanisms of the individual RNA and DNA building blocks are conserved and that dissociation of the phosphodiester backbone is primarily influenced by the adjacent 5'-nucleotide, resulting in the DNA-typical [a-base]- and w-ions and the RNA-typical c- and y-ions.
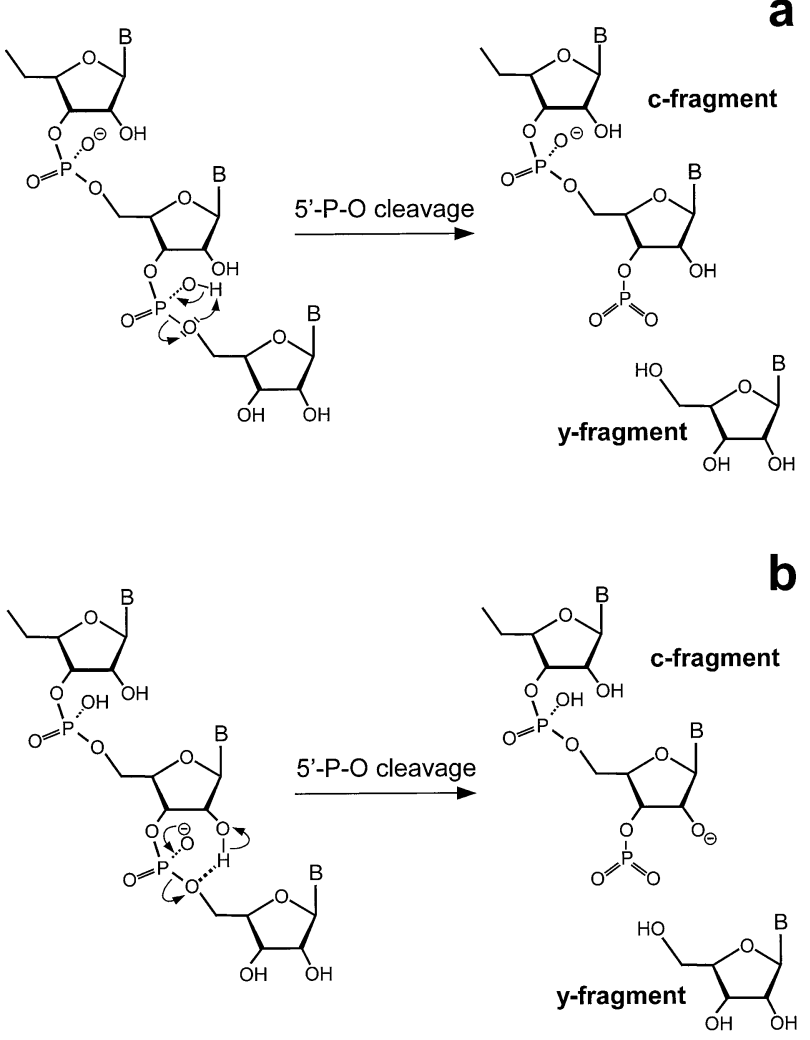

Scheme 1 Proposed fragmentation mechanism of RNA-oligonucleotides, resulting in c- and y-type fragments.

This work demonstrates that sequence information of RNA- and mixed-sequence RNA/DNA-oligonucleotides is readily obtained from the main fragment ion series. However, the formation of multiple fragment ion series does not only provide supporting sequence information, it also complicates spectral interpretation due to the presence of isobaric ions. The results emphasize the necessity of further detailed investigation of the mechanisms responsible for backbone dissociation and nucleobase loss in order to assign the various ion series. With this knowledge it should be feasible to extent the methodology to larger oligonucleotides.

\section{Acknowledgments}

The authors gratefully acknowledge the generous financial support from the Swiss National Science Foundation and the University of Bern. They also wish to thank Dr. S. König (Applied Biosystems, Rotkreuz, Switzerland) for technical support.

\section{References}

1. Moser, H. E.; Dervan, P. B. Sequence-Specific Cleavage of Double Helical DNA by Triple Helix Formation. Science 1987, 238, 645-650.

2. Nordhoff, E.; Kirpekar, F.; Roepstorff, P. Mass Spectrometry of Nucleic Acids. Mass Spectrom. Rev. 1996, 15, 67-138.

3. Limbach, P. A. Indirect Mass Spectrometric Methods for Characterizing and Sequencing Oligonucleotides. Mass Spectrom. Rev. 1996, 15, 297-336. 
4. Murray, K. K. DNA Sequencing by Mass Spectrometry. J. Mass Spectrom. 1996, 31, 1203-1215.

5. Burlingame, A. L.; Boyd, R. K.; Gaskell, S. J. Mass Spectrometry. Anal. Chem. 1998, 70, 647R-716R.

6. McLuckey, S. A.; Van Berkel, G. J.; Glish, G. L. Tandem Mass Spectrometry of Small, Multiply Charged Oligonucleotides. J. Am. Soc. Mass Spectrom. 1992, 3, 60-70.

7. McLuckey, S. A.; Habibi-Goudarzi, S. Ion Trap Tandem Mass Spectrometry Applied to Small Multiply Charged Oligonucleotides with a Modified Base. J. Am. Soc. Mass Spectrom. 1994, 5, 740-747.

8. McLuckey, S. A.; Habibi-Goudarzi, S. Decomposition of Multiply Charged Oligonucleotide Anions. J. Am. Chem. Soc. 1993, 115, 12085-12096.

9. Barry, J. P.; Vouros, P.; Van Schepdael, A.; Law, S.-J. Mass and Sequence Verification of Modified Oligonucleotides Using Electrospray Tandem Mass Spectrometry. J. Mass Spectrom. 1995, 30, 993-1006.

10. Little, D. P.; Aaserud, D. J.; Valaskovic, G. A.; McLafferty, F. W. Sequence Information from 42-108 mer DNAs (Complete for a 50 mer) by Tandem Mass Spectrometry. J. Am. Chem. Soc. 1996, 118, 9352-9359.

11. Marzilli, L. A.; Barry, J. A.; Sells, T.; Law, S.-J.; Vouros, P.; Harsch, A. Oligonucleotide Sequencing Using GuanineSpecific Methylation and Electrospray Ionization Ion Trap Mass Spectrometry. J. Mass Spectrom. 1999, 34, 276-280.

12. Christian, N. P.; Reilly, J. P.; Mokler, V. R.; Wincott, F. E.; Ellington, A. D. Elucidation of the Initial Step of Oligonucleotide Fragmentation in Matrix-Assisted Laser Desorption/ Ionization Using Modified Nucleic Acids. J. Am. Soc. Mass Spectrom. 2001, 12, 744-753.

13. Gross, J.; Leisner, A.; Hillenkamp, F.; Hahner, S.; Karas, M.; Schäfer, J.; Lützenkirchen, F.; Nordhoff, E. Investigations of the Metastable Decay of DNA under Ultraviolet MatrixAssisted Laser Desorption/Ionization Conditions with PostSource-Decay Analysis and Hydrogen/Deuterium Exchange. J. Am. Soc. Mass Spectrom. 1998, 9, 866-878.

14. Little, D. P.; Chorusch, R. A.; Speir, J. P.; Senko, M. W.; Kelleher, N. L.; McLafferty, F. W. Rapid Sequencing of Oligonucleotides by High-Resolution Mass Spectrometry. J. Am. Chem. Soc. 1994, 116, 4893-4897.

15. Cerny, R. L.; Tomer, K. B.; Gross, M. L.; Grotjahn, L. Fast Atom Bombardment Combined with Tandem Mass Spectrometry for Determining Structures of Small Oligonucleotides. Anal. Biochem. 1987, 165, 175-182.

16. Rodgers, M. T.; Campbell, S.; Marzluff, E. M.; Beauchamp, J. L. Low-Energy Collision-Induced Dissociation of Deprotonated Dinucleotides: Determination of the Energetically Favored Dissociation Pathways and the Relative Acidities of the Nucleic Acid Bases. Int. J. Mass Spectrom. Ion Processes 1994, 137, 121-149.

17. McLuckey, S. A.; Vaidyanathan, G.; Habibi-Goudarzi, S. Charged versus Neutral Nucleobase Loss from Multiply Charged Oligonucleotide Anions. J. Mass Spectrom. 1995, 30, 1222-1229.

18. Wang, Z.; Wan, K. X.; Ramanathan, R.; Taylor, J. S.; Gross, M. L. Structure and Fragmentation Mechanisms of Isomeric
T-Rich Oligodeoxynucleotides: A Comparison of Four Tandem Mass Spectrometric Methods. J. Am. Soc. Mass Spectrom. 1998, 9, 683-691.

19. Wan, K. X.; Gross, M. L. Fragmentation Mechanisms of Oligodeoxynucleotides: Effects of Replacing Phosphates with Methylphosphonates and Thymines with Other Bases in Trich Sequences. J. Am. Soc. Mass Spectrom. 2001, 12, 580-589.

20. Panico, M.; Sindona, G.; Uccella, N. Bioorganic Applications of Mass Spectrometry. 3. Fast-Atom-Bombardment-Induced Zwitterionic Oligonucleotide Quasimolecular Ions Sequenced by MS/MS. J. Am. Chem. Soc. 1983, 105, 5607-5610.

21. Gross, J.; Hillenkamp, F.; Wan, K. X.; Gross, M. L. Metastable Decay of Negatively Charged Oligodeoxynucleotides Analyzed With Ultraviolet Matrix-Assisted Laser Desorption/ Ionization Post-Source Decay and Deuterium Exchange. J. Am. Soc. Mass Spectrom. 2001, 12, 180-192.

22. Wan, K. X.; Gross, J.; Hillenkamp, F.; Gross, M. L. Fragmentation Mechanisms of Oligodeoxynucleotides Studied by H/D Exchange and Electrospray Ionization Tandem Mass Spectrometry. J. Am. Soc. Mass Spectrom. 2001, 12, 193-205.

23. Ni, J.; Pomerantz, S. C.; Rozenski, J.; Zhang, Y.; McClosky, J. A. Interpretation of Oligonucleotide Mass Spectra for Determination of Sequence Using Electrospray Ionization and Tandem Mass Spectrometry. Anal. Chem. 1996, 68, 1989-1999.

24. Tang, W.; Zhu, L.; Smith, L. M. Controlling DNA Fragmentation in MALDI-MS by Chemical Modification. Anal Chem. 1997, 69, 302-312.

25. Griffey, R. H.; Greig, M. J.; An, H.; Sasmor, H.; Manalili, S. Targeted Site-Specific Gas-Phase Cleavage of Oligoribonucleotides. Application in Mass Spectrometry-Based Identification of Ligand Binding Sites. J. Am. Chem. Soc. 1999, 121, $474-475$.

26. Kirpekar, F.; Krogh, T. N. RNA Fragmentation Studied in a Matrix-Assisted Laser Desorption/Ionization Tandem Quadrupole/Orthogonal Time-of-Flight Mass Spectrometer. Rapid Commun. Mass Spectrom. 2001, 15, 8-14.

27. Méndez-Bernal, E.; Leumann, C. J. Conformational Diversity versus Nucleic Acid Triplex Stability, a Combinatorial Study. J. Biol. Chem. 2001, 276, 35320-35327.

28. Users Manual 56-1111-56: Gene Assembler Special/4 Primers; Amersham Pharmacia Biotech: Piscataway, NJ;.

29. Greco, F.; Liguori, A.; Sindona, G.; Uccella, N. Gas-Phase Proton Affinity of Deoxyribonucleosides and Related Nucleobases by Fast Atom Bombardment Tandem Mass Spectrometry. J. Am. Chem. Soc. 1990, 112, 9092-9096.

30. Liguori, A.; Napoli, A.; Sindona, G. Survey of the Proton Affinities of Adenine, Cytosine, Thymine and Uracil Dideoxyribonucleosides, Deoxyribonucleosides and Ribonucleosides. J. Mass Spectrom. 2000, 35, 139-144.

31. Clauwaert, J.; Stockx, J. Interactions of Polynucleotides and Their Components: I. Dissociation Constants of the Bases and Their Derivatives. Z. Naturforsch. B 1968, 23, 25-30.

32. Bartlett, M. G.; McCloskey, J. A.; Manalili, S.; Griffey, R. H. The Effect of Backbone Charge on the Collision-Induced Dissociation of Oligonucleotides. J. Mass Spectrom. 1996, 31, 12771283. 